\title{
The Accuracy of Blood Pressure Monitoring Using the Senbiosys Ring: A Study on Patients Undergoing Coronary Angiography and Patients in the Intensive Care Unit
}

\section{Serj Haddad}

Senbiosys SA, Neuchatel

\section{Sara Schukraft}

University and Hospital of Fribourg

Assim Boukhayma

Senbiosys SA, Neuchatel

Anthony Barison

Senbiosys SA, Neuchatel

Yannick Faucherre

University and Hospital of Fribourg

Stéphane Cook

University and Hospital of Fribourg

Antonino Caizzone ( $\nabla$ antonino.caizzone@senbiosys.com )

Senbiosys SA, Neuchatel

\section{Research Article}

Keywords: patients, mmhg, values, undergoing, coronary, angiography

Posted Date: November 10th, 2021

DOI: https://doi.org/10.21203/rs.3.rs-1040431/v1

License: (c) (i) This work is licensed under a Creative Commons Attribution 4.0 International License.

Read Full License 


\title{
The Accuracy of Blood Pressure Monitoring Using the Senbiosys Ring: A Study on Patients Undergoing Coronary Angiography and Patients in the Intensive Care Unit
}

\author{
Serj Haddad ${ }^{1}$, Sara Schukraft ${ }^{2,+}$, Assim Boukhayma ${ }^{1}$, Anthony Barison ${ }^{1}$, Yannick \\ Faucherre $^{2}$, Stéphane Cook $^{2}$, and Antonino Caizzone ${ }^{1, *}$ \\ ${ }^{1}$ Senbiosys SA, Neuchâtel, 2000, Switzerland \\ ${ }^{2}$ Departement of Cardiology, University and Hospital of Fribourg, Fribourg, 1752, Switzerland \\ *antonino.caizzone@senbiosys.com \\ +Affiliation during the study
}

\begin{abstract}
The study NCT04379986 aims at evaluating the performance of the Senbiosys technology in monitoring blood pressure (BP) values for elderly patients: 25 patients undergoing coronary angiography, aged $68.92 \pm 6.41$ years, and 10 patients in the intensive care unit (ICU), aged $71.90 \pm 8.56$ years. The technology is based on pulse wave analysis (PWA) of low-power photoplethysmography (PPG) from the SBF2003 ring. The PPG recordings are processed by the PPG-based blood pressure monitoring (PPG-BPM) algorithm ${ }^{1}$ to generate systolic BP (SBP) and diastolic BP (DBP) estimates. For the SBP, the mean difference \pm standard deviation between the reference arterial line and the PPG-BPM values is $0 \pm 5.06 \mathrm{mmHg}$ for the patients undergoing coronary angiography and $0 \pm 7.46 \mathrm{mmHg}$ for the patients in the ICU. For the DBP, the mean difference \pm standard deviation between the reference arterial line and the PPG-BPM values is $0 \pm 1.85 \mathrm{mmHg}$ for the patients undergoing coronary angiography and $0 \pm 4.17 \mathrm{mmHg}$ for the patients in the ICU. The results show that the accuracy of our algorithm is within the $5 \pm 8 \mathrm{mmHg}$ ISO/ANSI/AAMI protocol requirement.
\end{abstract}

\section{Introduction}

During the last decade, a growing number of wearable devices evolved to provide accurate, inexpensive, and unobtrusive solutions to monitor vital signs using photoplethysmography (PPG). Hypertension, being a major contributing risk factor for heart failure, myocardial infarction, stroke, and chronic kidney disease ${ }^{2}$, and a major cause of premature death worldwide ${ }^{3}$, is an important vital that requires monitoring.

The arterial catheter is the gold standard to measure BP values continuously and accurately, however it is invasive and not suitable for daily ambulatory use ${ }^{4}$. On the other hand, the non-invasive cuff-based, auscultatory and oscillometric, BP monitoring is intermittent and uncomfortable because it occludes the blood flow in arteries ${ }^{5}$. Thus, it is important to have an unobtrusive wearable device that is able to monitor the BP variations.

Photoplethysmography (PPG), which utilizes a light-emitting diode (LED) to illuminate the skin and measures the power of either the transmitted or the reflected light, was suggested as a plausible technique for continuous BP estimation ${ }^{6,7}$. This optical solution measures the variations in the volume of the blood flow. It is deployed in most of the mainstream wearable devices and smart phones. Studies confirm the accuracy of PPG-based solutions to monitor various vital signs, such as heart rate $(\mathrm{HR})$ and heart rate variability $(\mathrm{HRV})^{8,9}$, stress levels ${ }^{10}$, irregularity in heart beats ${ }^{11,12}$, sleep quality ${ }^{13}$, respiratory rate ${ }^{14}$, etc. For BP monitoring, PPG-based methods rely on either pulse wave velocity (PWV) techniques ${ }^{15,16}$ or pulse wave analysis (PWA) methods ${ }^{17,18}$. Since the PWV techniques require the use of auxiliary sensors, such electrocardiography (ECG), it is impractical and thus less attractive. In this work, we focus on PPG-based BP estimation based on pulse wave analysis (PWA) technique which solely requires PPG signals acquired using the ultra low power SBF2003 ring.

In a previous study ${ }^{19}$, we show the accuracy of the PPG-BPM algorithm for continuous blood pressure monitoring using the ultra-low power Senbiosys ring SBF2003. The obtained results fulfilled the ISO/ANSI/AAMI protocol requirement. However, the study involved young and healthy subjects and the performance evaluation was done with respect to the cuff-based recordings.

The purpose of this study NCT04379986 is to evaluate the precision of the same solution to measure systolic blood pressure (SBP) and diastolic blood pressure (DBP) compared to invasive blood pressure (BP) measurements. The study is on elderly 
subjects from the cardiology and the intensive care units.

\section{Methods}

\section{Ethical Consent and Patient Inclusion}

Adult subjects admitted to the intensive care unit (ICU) with an arterial catheter in place and patients admitted to the cardiac catheterization laboratory at the University and Hospital of Fribourg are eligible for the study. Patients with myocardial infarction, atrial fibrillation, COVID-19 infection, intra-cardiac monitoring, and/or refusing or being unable to give informed consent are excluded. The study was in accordance with the Declaration of Helsinki and in compliance with the European Directive 93/42/EEC on medical devices and the international standard ISO 14155:2011. It was approved by the local ethics committee (Commission Cantonale (VD) d'éthique de la Recherche sur l'être humain, CH 1012 Lausanne Switzerland, CER-VD no. 2020-00996) and registered under number NCT04379986 at www.clinicaltrials.gov on May 8, 2020. Written informed consent was obtained from 25 patients aged 55 years or older who were undergoing coronary angiography and from 10 patients aged 59 years or older in the ICU at the University Hosptial of Fribourg.

\section{Patients Undergoing Coronary Angiography}

In the cardiac catheterization laboratory of the University and Hospital of Fribourg, 25 patients addressed for a coronary angiography are enrolled for the current study. Table 1 summarizes the statistics of the 25 patients.

Table 1. The statistics of the 25 patients undergoing coronary angiography.

\begin{tabular}{ll}
\hline Gender & \\
$\quad$ male:female & $15: 10$ \\
Age (years) & \\
$\quad$ Range & $55-79$ \\
$\quad$ Mean \pm Std. Dev. & $68.92 \pm 6.41$ \\
Body Mass Index $\left(\mathrm{kg} / \mathrm{m}^{2}\right)$ & \\
$\quad$ Range & $21.10-38.20$ \\
$\quad$ Mean \pm Std. Dev. & $29.10 \pm 4.57$ \\
\hline
\end{tabular}

\section{Patients in the ICU}

In the intensive care unit of the University and Hospital of Fribourg, 10 patients are enrolled for the current study. Table 2 summarizes the statistics of the 10 patients.

Table 2. The statistics of the 10 patients in the ICU.

\begin{tabular}{ll}
\hline $\begin{array}{l}\text { Gender } \\
\text { male:female }\end{array}$ & $8: 2$ \\
Age (years) & \\
$\quad$ Range & $59-83$ \\
$\quad$ Mean \pm Std. Dev. & $71.90 \pm 8.46$ \\
Body Mass Index $\left(\mathrm{kg} / \mathrm{m}^{2}\right)$ & \\
$\quad$ Range & $15.30-46.80$ \\
$\quad$ Mean \pm Std. Dev. & $27.32 \pm 8.02$ \\
\hline
\end{tabular}

\section{The investigational device}

The SBF2003, shown in Fig. 1, is a wearable ring that measures and streams the finger PPG signal. SBF2003 embeds a micro-power PPG sensor delivering high fidelity PPG signal with micro-power consumption at the level of the emitter and detector. The detector is a monolithic photonic sensor exploiting a CMOS image sensor process technology. Table 3 lists the high level specifications of the SBF2003 ring while further details on the PPG sensor technology are reported in ${ }^{20}$. In addition to the PPG sensor, SBF2003 embeds a power management unit exploiting coin batteries as well as a micro-controller unit 
ensuring the communication of the device with a user interface thanks to the Bluetooth low energy (BLE). The SBF2003 is controlled via BLE by the Senbiosys proprietary firmware installed on a laptop. Once the SBF2003 is correctly placed on the patient's index finger, it can record and transfer the PPG data via the BLE to the computer. The recorded PPG measurements are fed into the PPG-BPM algorithm that runs in offline mode to generate the SBP and the DBP values.

Table 3. The Specifications of the SBF2003.

\author{
Green light reflection PPG \\ Sampling Frequency of $122 \mathrm{~Hz}$ \\ Average LED current consumption of $120 \mu \mathrm{A}$ \\ Bluetooth Low Energy 5 (BLE5) \\ More than 8 hours of continuous recording \\ Battery capacity of $40 \mathrm{mAh}$ \\ Adjustable ring diameter between 16 and $24 \mathrm{~mm}$ \\ Weight of 12 grams \\ Made of biocompatible PA12
}

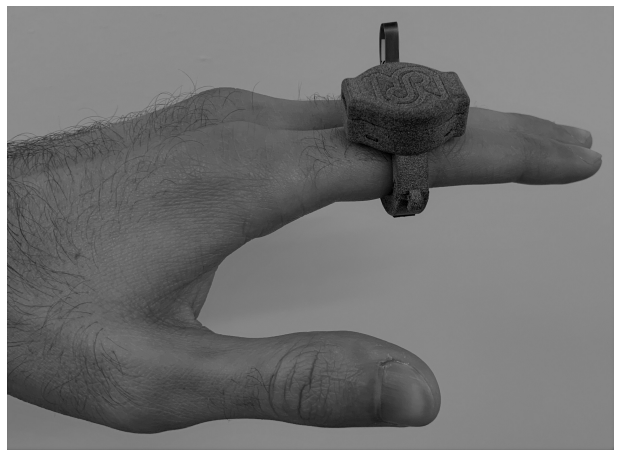

Figure 1. The SBF2003 on the index finger.

\title{
Study protocol and data acquisition \\ Study on Patients Undergoing Coronary Angiography
}

The investigator places the ring on the left hand - the contralateral side of the cardiac procedure - of the patient's index finger to avoid interference with the cardiac procedure. The device is placed prior to the placement of the sterile field. The arterial puncture is performed either via the radial or the femoral access. Intra-arterial BP measurements are recorded using a fluid-filled catheter. The study protocol involves three 3 minute measurements: 1) upon the positioning of the catheter in the aorta for an initial stable BP recording, 2) immediately after the administration of $300 \mu \mathrm{g}$ of nitroglycerin (NTG) (the administration of the nitroglycerin, which is a vasodilating agent, is a standard procedure during the coronary angiography), and 3 ) at the end of the coronary angiography for a final stable BP recording. The beat-to-beat reference SBP and DBP values are generated in offline mode using MATLAB ${ }^{\circledR}$ by detecting the peaks and the troughs, respectively, of the arterial blood pressure (ABP) signal. The ABP signal has a sampling frequency of $1 \mathrm{kHz}$.

\section{Study on Patients in the ICU}

The reference invasive blood pressure values are obtained by the Draeger Infinity ${ }^{\circledR} \mathrm{M} 540$ using a resistive strain gauge transducer. The reference SBP and DBP values are provided by the device itself at a rate of $1 / 60 \mathrm{~Hz}$ (one SBP and one DBP value every minute). The investigator places the ring on the index finger of the contralateral hand. The study protocol involves three 5 minute measurements during three separate periods of the day (in the morning, at noon, in the afternoon). Using three separate periods of 5 minute recordings each introduces variations in the blood pressure values.

\section{Data processing}

The data processing is done offline upon the completion of all the 35 recordings. Three of the coronary angiography recordings contained uninterrupted episodes of arrhythmia and were subsequently discarded. The remaining 32 recordings were processed 
by the Senbiosys proprietary beat-to-beat detection algorithm ${ }^{9,21}$ to identify the PPG pulses and generate signal quality indexes (SQI). The SQI index is a value between 0 and 1 that indicates the reliability of the beat estimation, with larger index values indicating higher reliability. The SQI value is based on: 1) the eligibility of the detected beat length, 2) how much the detected beat interval deviates from the previous beat intervals, 3) the skewness of the corresponding PPG pulse. It is important to have PPG pulses with high SQI values for a reliable and accurate pulse wave analysis (PWA).

The PPG pulses with high SQI values are eligible to be processed by the PWA block of the Senbiosys proprietary PPG-BPM algorithm ${ }^{1}$. The PWA block extracts several time-related and amplitude-related features, as proposed in the state-of-the-art, from the qualified PPG pulses. The extracted features are then mapped to two values using multiple linear regression (MLR) models. The two values generated by the MLR models represent the uncalibrated SBP and DBP estimates of the PPG-BPM algorithm corresponding to a specific PPG pulse.

The generated beat-to-beat BP estimates are then grouped into intervals/epochs of 10 seconds each. Epochs containing sufficient number of BP estimates, called clean epochs, are kept and the remaining epochs are discarded. Finally, the algorithm generates uncalibrated SBP and DBP estimates for each clean epoch using the beat-to-beat BP estimates available in the given epoch.

\section{Coronary Angiography Recordings}

The reference SBP and DBP values from the arterial line are averaged within each epoch. In this study, five of the remaining 22 recordings were discarded due to the absence of clean epochs. In total, the PPG-BPM algorithm generates 708 10-s clean epochs belonging to 17 patients. Refer to Table 4 for the statistics of the 17 participants and the reference BP data corresponding to the 70810 -s epochs.

Table 4. The statistics of the 708 10-s epochs from the 17 patients undergoing coronary angiography with adequate PPG data for analysis by the PPG-BPM algorithm.

\begin{tabular}{lc}
\hline Gender & \\
male:female & $11: 6$ \\
Age (years) & $55-79$ \\
$\quad$ Range & $70.53 \pm 6.36$ \\
Mean \pm Std. Dev. & \\
Body Mass Index $\left(\mathrm{kg} / \mathrm{m}^{2}\right)$ & $21.08-38.21$ \\
$\quad$ Range & $29.51 \pm 5.26$ \\
$\quad$ Mean \pm Std. Dev. & \\
Number of Clean Epochs Per Patient & $16-61$ \\
$\quad$ Range & $41.65 \pm 13.98$ \\
Mean \pm Std. Dev. & \\
SBP $(\mathrm{mmHg})$ & $83.90-192.97$ \\
$\quad$ Range & $134.87 \pm 18.31$ \\
Mean \pm Std. Dev. & \\
DBP (mmHg) & $42.86-89.07$ \\
$\quad$ Range & $63.01 \pm 10.00$ \\
Mean \pm Std. Dev. & \\
$\Delta$ SBP $(\mathrm{mmHg})$ & $37.44 \pm 15.07$ \\
Mean \pm Std. Dev. & \\
$\Delta$ DBP $(\mathrm{mmHg})$ & $11.40 \pm 4.60$ \\
$\quad$ Mean \pm Std. Dev. &
\end{tabular}

\section{ICU Recordings}

The reference SBP and DBP values from the arterial line are generated at a rate of 1/60 Hz (one SBP and one DBP value every minute) by the device to which the arterial line is connected. In total, we collect $171 \mathrm{SBP}$ and DBP measurements for our reference. On the other hand, the PPG-BPM algorithm generates 10-s clean epochs for all the 10 ICU recordings. Note that our algorithm was capable of generating BP estimates for all the reference measurements. Refer to Table 5 for the statistics of the reference BP data. 
Table 5. The statistics of the 171 reference BP measurements from the 10 patients in the ICU.

\begin{tabular}{lc}
\hline SBP $(\mathrm{mmHg})$ & \\
Range & $85-157$ \\
Mean \pm Std. Dev. & $121.96 \pm 18.29$ \\
DBP $(\mathrm{mmHg})$ & \\
$\quad$ Range & $39-74$ \\
Mean \pm Std. Dev. & $55.63 \pm 9.05$ \\
$\Delta$ SBP $(\mathrm{mmHg})$ & \\
$\quad$ Mean \pm Std. Dev. & $24.20 \pm 11.26$ \\
$\Delta$ DBP $(\mathrm{mmHg})$ & $13.90 \pm 5.55$ \\
$\quad$ Mean \pm Std. Dev. & \\
\hline
\end{tabular}

\section{Pulse wave analysis}

The technical details of the PPG-BPM algorithm that performs the pulse wave analysis to generate SBP and DBP estimates are presented in ${ }^{1}$. In what follows, we present a brief description of the algorithm which includes: 1) pre-processing of the PPG signal and beat detection, 2) extraction of the relevant features from the processed PPG signal and mapping of these features to pressure values.

\section{Pre-processing and beat detection}

The different stages of the pre-processing and beat detection sub-blocks are illustrated in Fig. 2. We start by denoising the input PPG signal acquired using the Senbiosys ring (see the first waveform from top in Fig. 2). For this purpose, we use the Savitzky-Golay smoothing filter. Then, the PPG pulses are identified using the beat-to-beat detection algorithm presented in ${ }^{21}$. As mentioned, each detected beat is associated with an SQI value. The SQI values represent the quality of the PPG signal and are based on the skewness and the variation of the inter-beat intervals (IBI) of the PPG pulses (see the second waveform from top in Fig. 2).

The beat detection algorithm detects the peaks of the first derivative of the PPG signal which represent the maximum up-slopes of the PPG signal. For this reason, we further identify the troughs by finding the global minimums between the consecutive maximum up-slope points. After identifying the troughs, the PPG signal is detrended and normalized to amplitude one (see the third waveform from top in Fig. 2).

PPG pulses recorded on flexible arteries (young and healthy subjects) have very clear distinct features with all the necessary fiduciary points. On the contrary, pulses recorded from stiffer arteries (elderly and unhealthy subjects) show fewer or less distinctive features, or may even lack the fiduciary points and show no features at all. Given that the current study is held on critically ill elderly patients, the acquired PPG data are very challenging and show no visually traceable features. This means the feature extraction sub-block of our algorithm will simply discard all the recorded data because they lack the necessary features required to generate pressure values. For this reason, the PPG signal undergoes another stage of transformation by a low-pass second order type I Chebyshev filter whose pass-band ripple introduces artificial fiduciary points. The filtered signal is then re-normalized and forwarded to the feature extraction and mapping sub-blocks (see the last waveform in Fig. 2).

\section{Feature extraction and mapping}

In Fig. 3, we illustrate the feature extraction and mapping sub-blocks of the PPG-BPM algorithm. The feature extraction sub-block discards the PPG pulses that have low SQI values. Afterwards, it identifies the fiduciary points of the remaining PPG pulses. These features are based on the following fiduciary points: the maximum slope on the up-rise, the systolic peak, the dicrotic notch, the inflection point, the diastolic peak, and the five acceleration PPG (APPG - the second derivative of the PPG) waves $a, b, c, d$, and $e$. Using the identified fiduciary points, several time-related and amplitude-related features, which are widely used in the literature ${ }^{18}$, are derived. Finally, these features are mapped to pressure values using two multi-regression models, one to generate SBP values and another to generate DBP values. Given that the generated BP estimates are based on the volumetric changes in the blood flow and not on a real pressure signal, they require further calibration and thus said to be uncalibrated BP estimates.

\section{Calibration}

The SBP and the DBP estimates from the algorithm are uncalibrated. To calibrate the BP estimates, we adopt the zero-mean calibration ${ }^{1}$. For each recording, the zero-mean calibration introduces SBP and DBP offsets so that the mean errors with respect to the reference SBP and DBP values are equal to zero. 


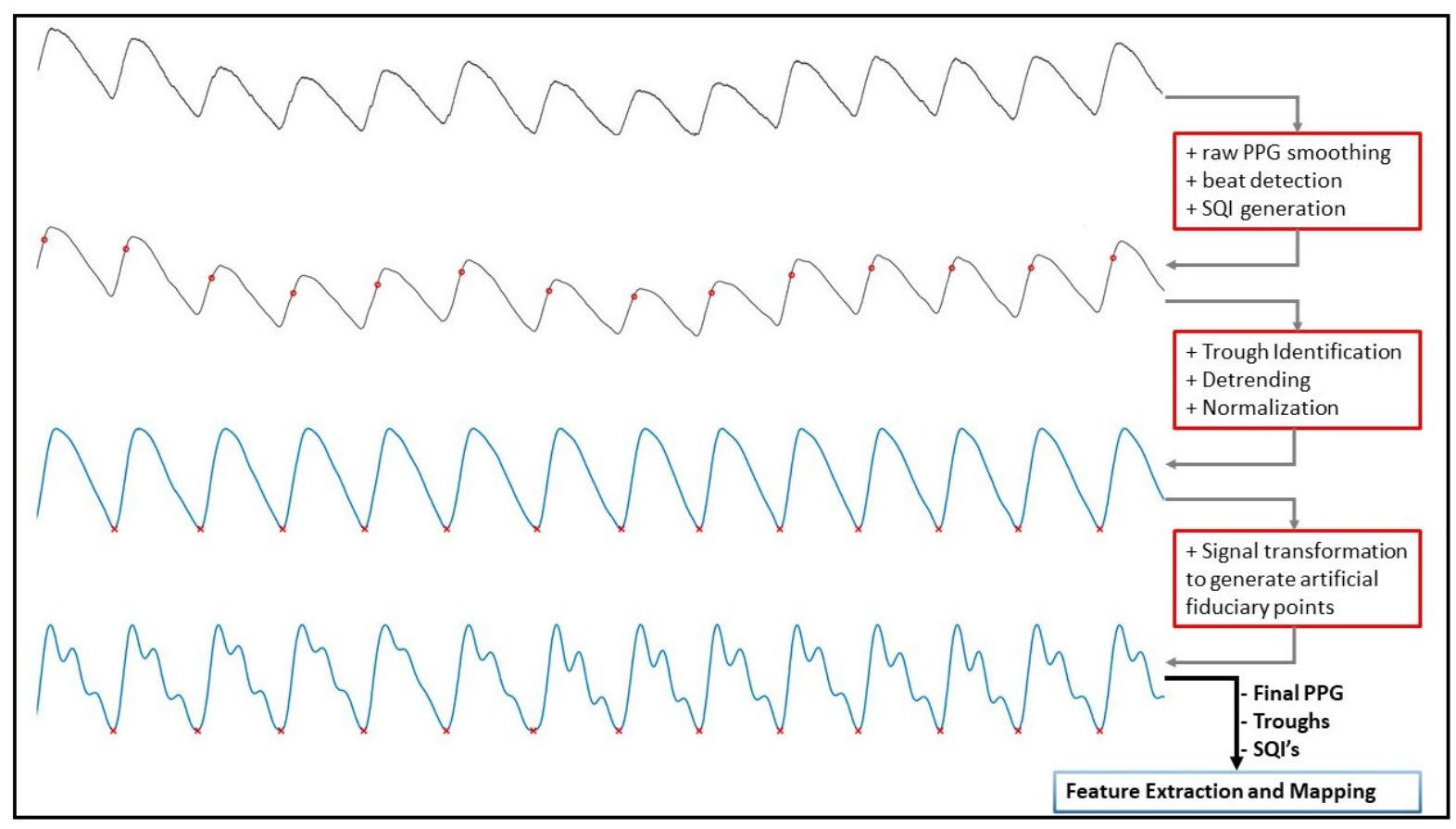

Figure 2. Pre-processing and beat detection sub-blocks of the PPG-BPM algorithm.

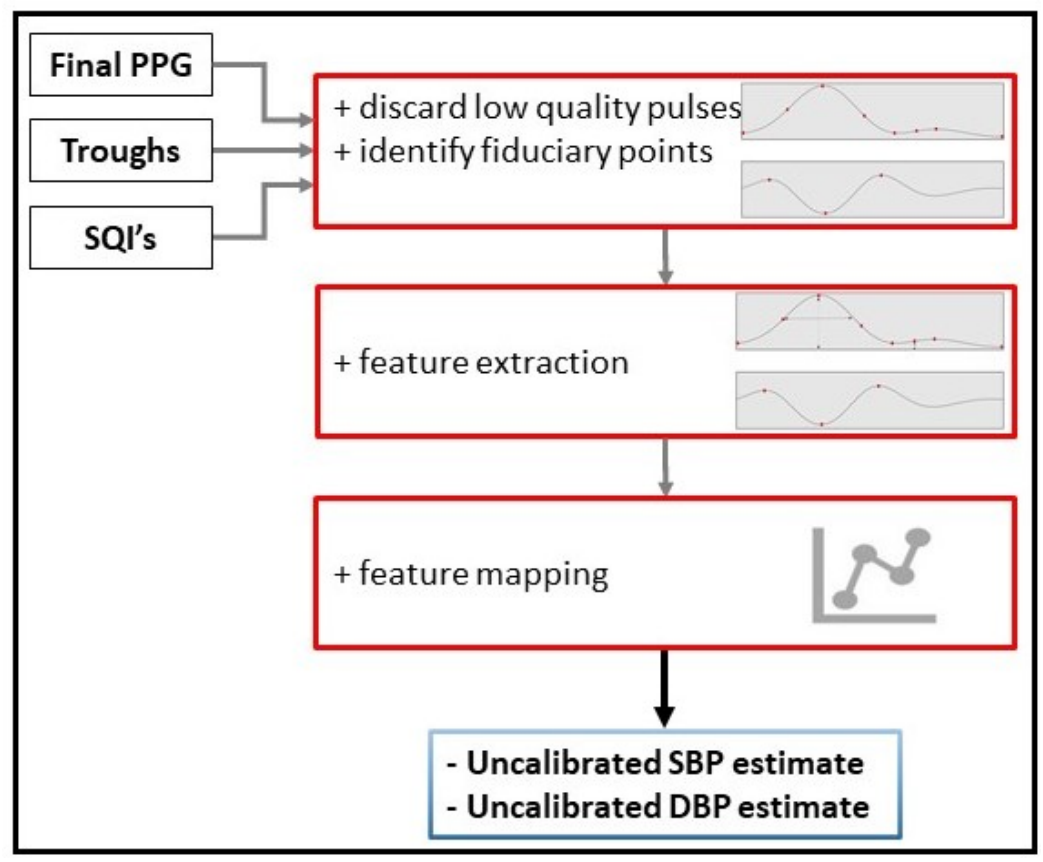

Figure 3. Feature extraction and mapping sub-blocks of the PPG-BPM algorithm.

\section{Performance Evaluation}

The performance evaluation of the investigational device shows that the accuracy achieved in estimating the SBP and the DBP is within the $5 \pm 8 \mathrm{mmHg}$ ISO/ANSI/AAMI protocol requirements.

\section{Results of the Coronary Angiography Recordings}

The performance evaluation of the investigational device is summarized in Table 6. The obtained results confirm the accuracy of the Senbiosys solution in estimating both SBP and DBP values with respective error standard deviation values of $5.06 \mathrm{mmHg}$ 
and $2.45 \mathrm{mmHg}$. The results also show that more than $94 \%$ of the SBP estimates and $99 \%$ of the DBP estimates have an absolute error of less than $10 \mathrm{mmHg}$ compared to the invasive measurements.

Table 6. Overall performance evaluation over the 708 coronary angiography measurements.

\begin{tabular}{lll}
\hline & SBP & DBP \\
\hline MAE $(\mathrm{mmHg})$ & 3.81 & 1.85 \\
ME $(\mathrm{mmHg})$ & 0 & 0 \\
SDE $(\mathrm{mmHg})$ & 5.06 & 2.45 \\
$\leq 5 \mathrm{mmHg}(\%)$ & 71.61 & 94.34 \\
$\leq 10 \mathrm{mmHg}(\%)$ & 94.77 & 99.86 \\
$\leq 15 \mathrm{mmHg}(\%)$ & 98.31 & 100 \\
Pearson's $r$ & 0.96 & 0.97 \\
\hline
\end{tabular}

In Fig. 4, we illustrate the high correlation between the PPG-based BP values generated by the PPG-BPM algorithm and the corresponding arterial BP values: $r$ values of 0.96 and $0.97, p<.001$, for SBP and DBP values, respectively. In the same figures, we show the Bland-Altman plots, where the $95 \%$ limits of agreement are found to be $\{-9.92,+9.92\} \mathrm{mmHg}$ and $\{-4.80,+4.80\} \mathrm{mmHg}$ for the SBP and the DBP, respectively.

\section{Results of the ICU Recordings}

The performance evaluation of the investigational device is summarized in Table 7. The obtained results confirm the accuracy of the Senbiosys solution in estimating both SBP and DBP values with a respective error standard deviation of $7.46 \mathrm{mmHg}$ and $4.17 \mathrm{mmHg}$. The results also show that more than $81 \%$ of the SBP estimates and $98 \%$ of the DBP estimates have an absolute error of less than $10 \mathrm{mmHg}$ compared to the invasive measurements.

In Fig. 5, we illustrate the high correlation between the PPG-based BP values generated by the PPG-BPM algorithm and the corresponding arterial BP values: $r$ values of 0.91 and $0.89, p<.001$, for SBP and DBP values, respectively. In the same figures, we show the Bland-Altman plots, where the $95 \%$ limits of agreement are found to be $\{-14.62,+14.62\} \mathrm{mmHg}$ and $\{-8.17,+8.17\} \mathrm{mmHg}$ for the SBP and the DBP, respectively.

\section{Discussion}

$\mathrm{In}^{22}$, while the authors agree that cuffless wearable devices are attractive and promising, they validly question the evaluation scheme followed in many publications presenting data on the accuracy of the BP measurement devices leading to imprecise conclusions. In this section, we identify the limitations of the current study and propose a new evaluation scheme that takes into consideration these limitations. Accordingly, we revisit our results to provide an unbiased evaluation of our solution.

Table 7. Overall performance evaluation over the 171 ICU measurements.

\begin{tabular}{lll}
\hline & SBP & DBP \\
\hline MAE $(\mathrm{mmHg})$ & 5.67 & 3.13 \\
ME (mmHg) & 0 & 0 \\
SDE $(\mathrm{mmHg})$ & 7.46 & 4.17 \\
$\leq 5 \mathrm{mmHg}(\%)$ & 57.31 & 75.44 \\
$\leq 10 \mathrm{mmHg}(\%)$ & 81.29 & 98.83 \\
$\leq 15 \mathrm{mmHg}(\%)$ & 93.58 & 100 \\
Pearson's $r$ & 0.91 & 0.89 \\
\hline
\end{tabular}




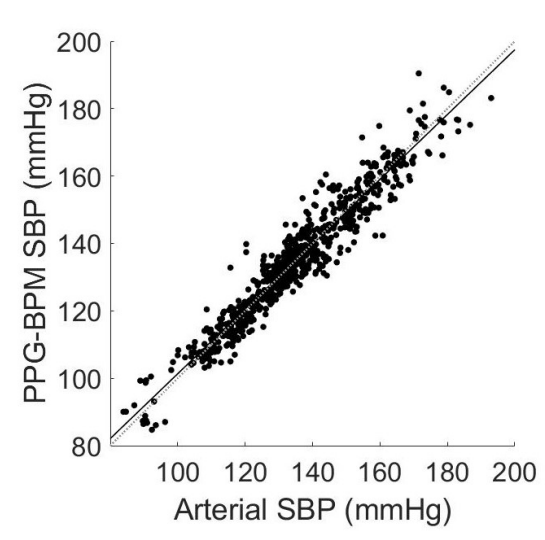

(a)

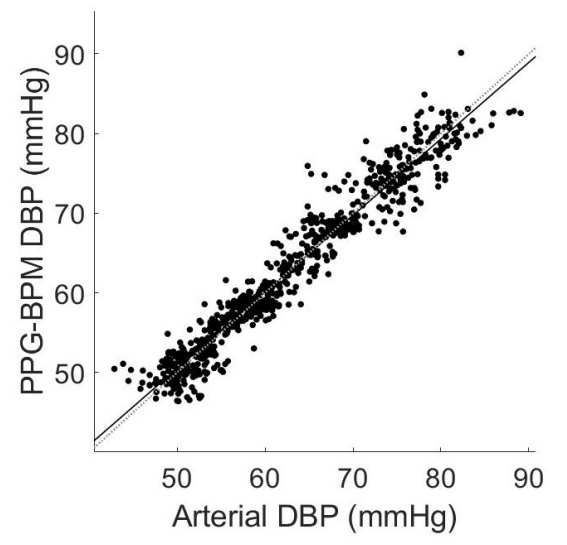

(c)

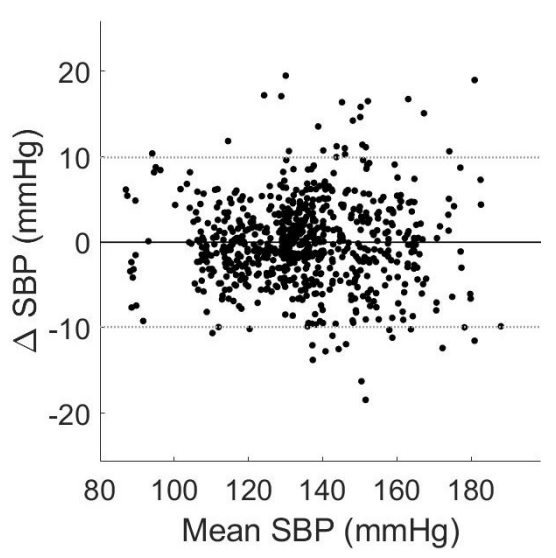

(b)

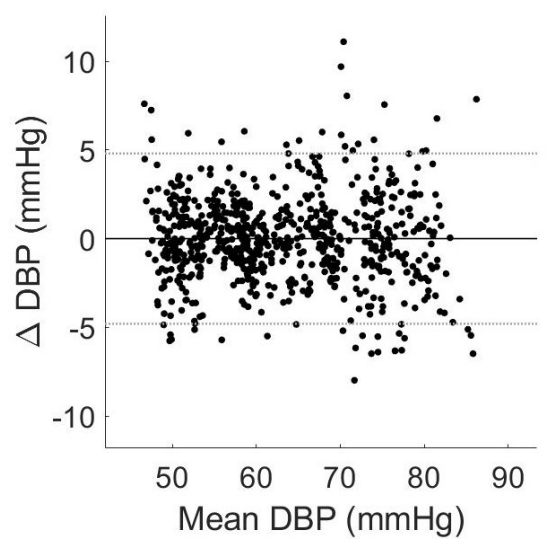

(d)

Figure 4. The outcome of the study on patients undergoing coronary angiography. (a) R-plot for the SBP values with Pearson correlation coefficient $r=0.96$. (b) Bland-Altman with $95 \%$ confidence interval $\mu \pm 1.96 \sigma=\{-9.92,9.92\} \mathrm{mmHg}$. (c) R-plot for the DBP values with Pearson correlation coefficient $r=0.97$. (d) Bland-Altman plot shows the 95\% confidence interval $\mu \pm 1.96 \sigma=\{-4.80,4.80\} \mathrm{mmHg}$.

\section{Limitations}

\section{Calibration}

One of the limitations of the study is the zero-mean calibration, which introduces SBP and DBP offsets to force the mean errors to zero. The reason behind adopting the zero-mean calibration scheme is that the arterial line provides continuous BP measurements allowing to track all the BP variations. Given that the current study involves large BP (especially SBP) fluctuations due to the administration of NTG and the fact that the measurements are done during coronary angiography, the main goal is to show that the BP estimates generated by the PPG-BPM algorithm accurately track the given variations. Note that using a starting-point calibration scheme ${ }^{1}$-this approach introduces SBP and DBP offsets for each subject based on the first reference SBP and DBP measurements- results in a similar performance as the zero-mean calibration.

\section{Limited DBP Variations}

For the study on patients undergoing coronary angiography, the total duration of each recording is 9 minutes (this is independent of the duration of the procedure which is 40 to 60 minutes): 6 minutes in the beginning of the procedure ( 3 minutes before and 3 minutes after the administration of NTG) and 3 minutes in the end of the procedure. In general, the SBP values are high during the first 3 minute interval. Upon the administration of the NTG (during the second 3 minute interval), the SBP values drop immediately. Finally, the SBP values stabilize and slightly increase to normal values in the last 3 minute interval of the procedure. For this reason, one can explain the large $\Delta \mathrm{SBP}$ values $(37.44 \pm 15.07 \mathrm{mmHg})$, as shown in Table 4. However, this is not the case for the DBP values, which experience smaller variations per recording ( $\triangle \mathrm{DBP}$ of $11.40 \pm 4.60 \mathrm{mmHg}$ ).

On the other hand, for the study on patients in the ICU, the duration of each recording is 15 minutes divided into three separate 5 minutes recordings with at least a break of two hours between them. This protocol introduces a slightly larger 


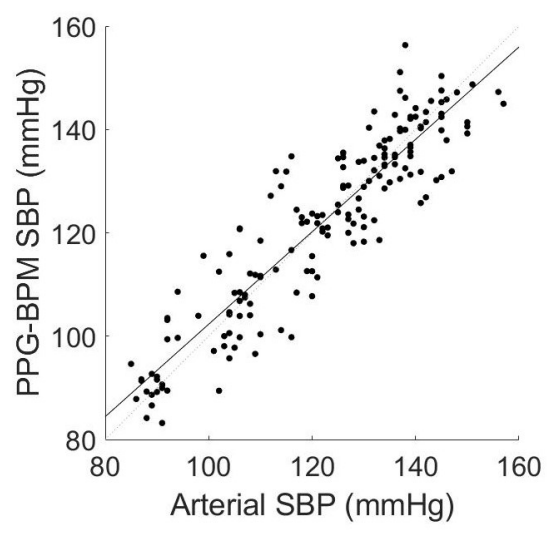

(a)

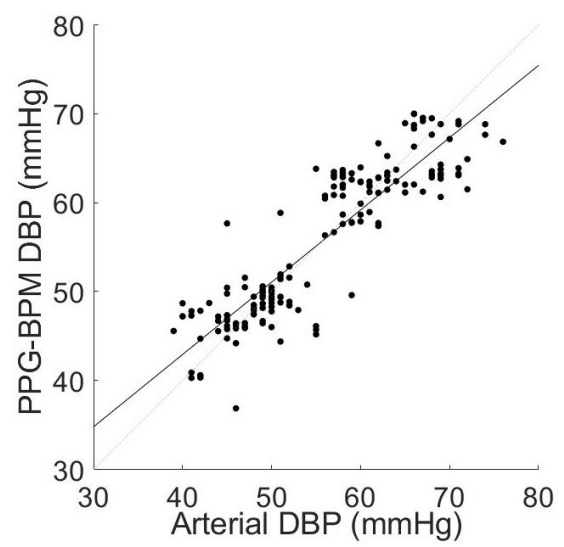

(c)

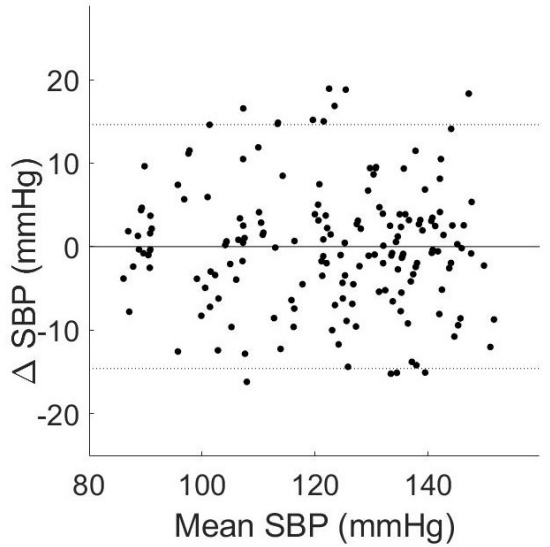

(b)

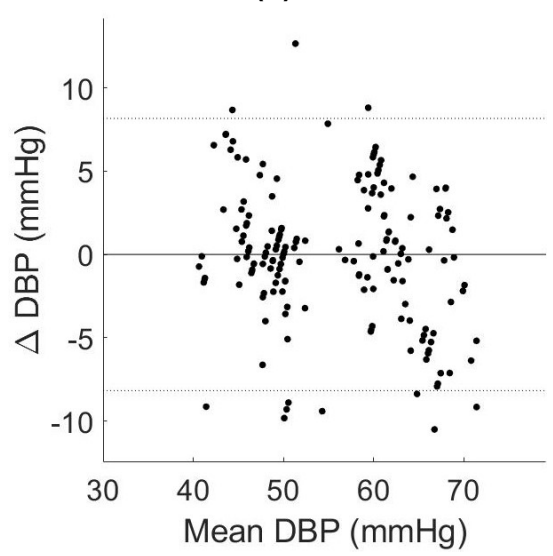

(d)

Figure 5. The outcome of the study on patients in the ICU. (a) R-plot for the SBP values with Pearson correlation coefficient $r=0.91$. (b) Bland-Altman with 95\% confidence interval $\mu \pm 1.96 \sigma=\{-14.62,14.62\} \mathrm{mmHg}$. (c) R-plot for the DBP values with Pearson correlation coefficient $r=0.89$. (d) Bland-Altman plot shows the $95 \%$ confidence interval $\mu \pm 1.96 \sigma=\{-8.17,8.17\} \mathrm{mmHg}$.

variation in the DBP values compared to the coronary angiography recordings.

\section{Proposition}

\section{New Evaluation Scheme}

It may be the case that the SBP and DBP offsets used to calibrate the output of the PPG-BPM algorithm are the reason behind the good performance. In other words, it may be that even if we solely use these offsets values as our BP estimates, the values of the performance metrics would still be acceptable. For this reason, we propose a new evaluation scheme that identifies the relative performance of our proposed BP measurement technique with respect to the offset BP values used for calibration.

\section{New Results}

The relative performance of the PPG-BPM algorithm on the coronary angiography measurements is presented in Table 8 . The first observation is that all mean error values are equal to zero. This is because we adopted the zero-mean calibration scheme where the offset BP values are equal to mean reference BP values. The second observation is that using the DBP offset values as our estimates ensures a good performance. For this reason, the performance metrics of the DBP estimates of the PPG-BPM algorithm are only negligibly better than that of the DBP offsets. This is due to the limited DBP variations, which is, as we already discussed, one of the limitations of this study. On the other hand, the SBP estimates of the PPG-BPM algorithm are significantly better than the SBP offsets. This means that the offsets values used to calibrate the SBP estimates of our algorithm are not the reason behind its good performance. 
Table 8. Relative performance evaluation over the 708 coronary angiography measurements.

\begin{tabular}{lllll}
\hline & SBP offset & PPG-BPM SBP & DBP offset & PPG-BPM DBP \\
\hline MAE $(\mathrm{mmHg})$ & 8.83 & 3.81 & 2.22 & 1.85 \\
$\mathrm{ME}(\mathrm{mmHg})$ & 0 & 0 & 0 & 0 \\
$\mathrm{SDE}(\mathrm{mmHg})$ & 11.11 & 5.06 & 2.98 & 2.45 \\
$\leq 5 \mathrm{mmHg}(\%)$ & 32.63 & 71.61 & 91.86 & 94.34 \\
$\leq 10 \mathrm{mmHg}(\%)$ & 63.98 & 94.77 & 99.31 & 99.86 \\
$\leq 15 \mathrm{mmHg}(\%)$ & 83.05 & 98.31 & 99.72 & 100 \\
Pearson's $r$ & 0.79 & 0.96 & 0.95 & 0.97 \\
\hline
\end{tabular}

\section{Conclusion}

The goal of this study is to evaluate the accuracy of the SBP and DBP estimates generated by the PPG-BPM algorithm using the PPG from the SBF2003 ring. The performance of the technology is evaluated on: 1) coronary angiography patients and this involves the administration of NTG, 2) intensive care unit patients during different periods of the day. The obtained results confirm the ability of the Senbiosys BP monitoring solution to accurately and continuously estimate the SBP and the DBP values. The standard deviation and the mean absolute of the errors (for both the systolic and the diastolic blood pressure values) between the PPG estimates and the invasive measurements are less than $8 \mathrm{mmHg}$ and $6 \mathrm{mmHg}$, respectively. Moreover, more than $80 \%$ of the estimates have an absolute error of less than $10 \mathrm{mmHg}$ and more than $90 \%$ of the estimates have an absolute error of less than $15 \mathrm{mmHg}$. In ${ }^{19}$, we showed the feasibility of accurately (with respect to cuff based measurements) monitoring BP values by applying the PPG-BPM algorithm on the optical data acquired from healthy and young subjects using the ultra-low power Senbiosys ring SBF2003. In this work, we complement the previously obtained results by clinical trials to evaluate the performance of the PPG-BPM algorithm with respect to the invasive arterial line and for critically ill elderly subjects.

\section{References}

1. Haddad, S., Boukhayma, A. \& Caizzone, A. Continuous ppg-based blood pressure monitoring using multi-linear regression (https://arxiv.org/abs/2011.02231).

2. Force, U. P. S. T. Screening for hypertension in adults: Us preventive services task force reaffirmation recommendation statement. JAMA 325, 1650-1656 (2021).

3. WHO. Hypertension (2019). Https://www.who.int/news-room/fact-sheets/detail/hypertension.

4. Davies, R. J. O., Jenkins, N. E. \& Stradling, J. R. Effect of measuring ambulatory blood presure on sleep and on blood pressure during sleep. British Medical Journal 308, 820-23 (1994).

5. Park, A. K., Menard, S. W. \& Yuan, C. Comparison of auscultatory and oscillometric blood pressures. Archives of Pediatrics \& Adolescent Medicine 155, 50-53 (2001).

6. Teng, X. F. \& Zhang, Y. T. Continuous and noninvasive estimation of arterial blood pressure using a photoplethysmographic approach. In Proceedings of the 25th Annual International Conference of the IEEE Engineering in Medicine and Biology Society (IEEE Cat. No.03CH37439), vol. 4, 3153-3156 (2003).

7. Choudhury, A. D., Banerjee, R., Sinha, A. \& Kundu, S. Estimating blood pressure using windkessel model on photoplethysmogram. In 2014 36th Annual International Conference of the IEEE Engineering in Medicine and Biology Society, 4567-4570 (2014).

8. Acharya, U. R., Joseph, K. P., Kannathal, N., Lim, C. M. \& Suri, J. S. Heart rate variability: a review. In Medical \& Biological Engineering \& Computing, vol. 44, 1031-1051 (2006).

9. Haddad, S., Boukhayma, A. \& Caizzone, A. Ear and finger ppg wearables for night and day beat-to-beat interval detection. In International Conference of the IEEE Engineering in Medicine and Biology Society (EMBC) (2021).

10. Sztajzel, J. Heart rate variability: a noninvasive electrocardiographic method to measure the autonomic nervous system. vol. 134(35-36), 514-522 (Swiss Med Wkly, 2004). 
11. Haddad, S. et al. Ectopic beat-detection from wrist optical signals for sinus rhythm and atrial fibrillation subjects. In $X V$ Mediterranean Conference on Medical and Biological Engineering and Computing - MEDICON, 150-158 (2019).

12. Tarniceriu, A. et al. Performance of wrist photoplethysmography in monitoring atrial fibrillation in post cardiac surgery patients. In 2019 Computing in Cardiology (CinC), 1-4 (2019).

13. Renevey, P. et al. Optical wrist-worn device for sleep monitoring. In EMBEC \& NBC 2017, 615-618 (Springer Singapore, Singapore, 2018).

14. Haddad, S., Boukhayma, A. \& Caizzone, A. Ppg-based respiratory rate monitoring using hybrid vote-aggregate fusion technique. In International Conference of the IEEE Engineering in Medicine and Biology Society $(E M B C)(2021)$.

15. Choi, Y., Zhang, Q. \& Ko, S. Noninvasive cuffless blood pressure estimation using pulse transit time and hilbert-huang transform. Computers and Electrical Engineering 39, 103-111 (2013).

16. Liang, Y. et al. How effective is pulse arrival time for evaluating blood pressure? challenges and recommendations from a study using the mimic database. Clinical Medicine 8 (2019).

17. Atomi, K., Kawanaka, H., Bhuiyan, S. \& Oguri, K. Cuffless blood pressure estimation based on data-oriented continuous health monitoring system. In Computational and Mathematical Methods in Medicine, vol. 2017 (2017).

18. Shimazaki, S., Bhuiyan, S., Kawanaka, H. \& Oguri, K. Features extraction for cuffless blood pressure estimation by autoencoder from photoplethysmography. In International Conference of the IEEE Engineering in Medicine and Biology Society (EMBC), 2857-2860 (2018).

19. Haddad, S. et al. Photoplethysmography based blood pressure monitoring using the senbiosys ring. In International Conference of the IEEE Engineering in Medicine and Biology Society (EMBC) (2021).

20. Boukhayma, A., Barison, A., Haddad, S. \& Caizzone, A. Ring-embedded micro-power mm-sized optical sensor for accurate heart beat monitoring. IEEE Access 9, 127217-127225 (2021).

21. Haddad, S., Boukhayma, A. \& Caizzone, A. Beat-to-beat detection accuracy using the ultra low power senbiosys ppg sensor. In 8th European Medical and Biological Engineering Conference (EMBEC 2020), 178-188 (Springer, 2020).

22. Mukkamala, R. et al. Evaluation of the accuracy of cuffless blood pressure measurement devices: Challenges and proposals. Hypertension 78, 1161-1167 (2021).

\section{Acknowledgements}

The authors would like to thank the Cardiology Care Unit (CCU) and the Intensive Care Unit (ICU) teams in the University and Hospital of Fribourg. The clinical trial is funded by the Fonds Scientifique Cardiovasculaire Fribourg.

\section{Author contributions statement}

S.H.: This author helped for signal processing, statistical analysis, data interpretation, and manuscript preparation. He has approved the final version of the manuscript. S.S.: This author helped for study design, patient recruitment, data acquisition, and manuscript reviewing. She has approved the final version of the manuscript. A.B.: This author helped for study design, data acquisition, supervision of the engineering part of the study, and manuscript reviewing. He has approved the final version of the manuscript. A.B.: This author helped for design of the ring, data acquisition with a focus on the engineering part, and manuscript reviewing. He has approved the final version of the manuscript. Y.F.: This author helped for patient recruitment, data acquisition, and manuscript reviewing. He has approved the final version of the manuscript. S. C.: This author helped for study design, supervision of the clinical part of the study, patient recruitment, and manuscript reviewing. He has approved the final version of the manuscript. A. C.: This author helped for study design, supervision of the technical and the engineering parts of the study, data acquisition, and manuscript reviewing. He has approved the final version of the manuscript.

\section{Additional information}

There are no conflicts of interest. 